\section{EL PROBLEMA DEL DÉFICIT EN LOS MODELOS DEMOCRÁTICOS DE DIVULGACIÓN CIENTÍFICA}

\author{
Jorge M. Escobar \\ Instituto Tecnológico Metropolitano (Medellín, Colombia) \\ ORCID iD: http://orcid.org/0000-0003-3785-3114 \\ jorgeescobar@itm.edu.co
}

Cómo citar este artículo/Citation: Escobar, J. M. (2017). El problema del déficit en los modelos democráticos de divulgación científica. Arbor, 193 (785): a407. doi: http://dx.doi. org/10.3989/arbor.2017.785n3012

Recibido: 12 febrero 2016. Aceptado: 2 febrero 2017.

RESUMEN: Existe una tendencia en los estudios CTS contemporáneos a contraponer modelos deficitarios y modelos democráticos de divulgación científica. Esta contraposición suele apoyarse en lo que se conoce como el nuevo contrato social sobre ciencia y tecnología, y puede formularse así: mientras el viejo contrato social implicaba un modelo deficitario de divulgación científica, el nuevo contrato social implica un modelo democrático, que no es deficitario. En este artículo, defiendo la tesis de que dicha contraposición está mal fundada por dos razones. En primer lugar, el nuevo contrato social es compatible con diversos modelos democráticos de divulgación científica, y estos modelos no son compatibles entre sí necesariamente. En segundo lugar, algunos de estos modelos democráticos pueden calificarse también como deficitarios. Esto deja abierta la pregunta de cómo entender la divulgación científica en el marco del nuevo contrato social sobre ciencia y tecnología.

PALABRAS CLAVE: divulgación científica; modelo democrático de divulgación científica; déficit epistémico; déficit político.

\section{THE DEFICIT PROBLEM IN THE DEMOCRATIC MODELS OF SCIENCE POPULARIZATION}

Copyright: (C) 2017 CSIC. Este es un artículo de acceso abierto distribuido bajo los términos de la licencia Creative Commons Attribution (CC BY) España 3.0.
ABSTRACT: There is a tendency in contemporary STS studies to oppose deficit models and democratic models of science popularization. This opposition usually relies on a so-called new social contract for science and technology and can be formulated as follows: whereas the old social contract implied a deficit model of science popularization, the new social contract implies a democratic and not a deficit model. In this article, I defend the thesis that such an opposition is ill founded for two reasons. In the first place, the new social contract is compatible with different democratic models of science popularization, and these models are not necessarily compatible among themselves. In the second place, some of these democratic models can also be described as deficit models. In this sense, the question about how to understand science popularization in the framework of the new social contract for science and technology remains unanswered.

KEYWORDS: science popularization; democratic model of science popularization; epistemic deficit; political deficit. 


\section{INTRODUCCIÓN}

Parece claro que una aproximación comprehensiva a la historia del siglo XX resulta incompleta si se ignora el rol de la ciencia y la tecnología en él. No es exagerado afirmar que estas tuvieron un papel preponderante en la consolidación de los estados nacionales durante ese siglo, debido a su papel en eventos como las dos primeras guerras mundiales, conocidas como guerra de los químicos y guerra de los físicos respectivamente, y en la guerra fría (Kevles, 1995). La ciencia y la tecnología multiplicaron la capacidad destructiva de los seres humanos respecto a ellos mismos y al planeta en general, y como consecuencia generaron serias dudas acerca de uno de los ideales fundamentales del mundo moderno: que el conocimiento científico y tecnológico es necesariamente beneficioso para la humanidad, y por ello los estados deben hacer lo posible para promoverlo y desarrollarlo en sus sociedades y entre sus ciudadanos.

El escepticismo sobre este ideal llevó a buscar nuevos medios para establecer relaciones más estrechas y de mayor confianza entre ciencia, tecnología y sociedad. El argumento principal es que, al menos en los estados democráticos, los ciudadanos no solo deben participar, sino que tienen derecho a participar, en ciencia y tecnología, pues son asuntos que les afectan directa e indirectamente. Pero para que esto pueda suceder, esos mismos ciudadanos deben estar mejor informados sobre ciencia y tecnología. De otro modo, su participación resultaría inocua.

Para garantizar tal participación y la democratización asociada con ella, en las últimas décadas se ha empezado a hablar, especialmente a partir de la Conferencia Mundial sobre Ciencia de Budapest en 1999 (Cetto, 2000), acerca de la necesidad de implementar un nuevo contrato social sobre la ciencia y la tecnología que reemplace al viejo contrato social (Lubchenco, 1998; López Cerezo, Méndez Sanz y Todt, 1998; Gibbons, 1999; Guston, 2000; Olivé, 2003; Lozano, 2005). Olivé caracteriza estos dos contratos de la siguiente manera.

En el viejo contrato social, que suele asociarse con el informe Science, The Endless Frontier, presentado por Vannevar Bush al presidente de los Estados Unidos en 1945,

el sistema científico recibe apoyo de la sociedad por medio del Estado -especialmente para la investigación básica, cuyos resultados difícilmente tienen un valor de mercado-, se mantiene autónomo y relativamente aislado, es decir, fija sus propias reglas y metas y, a la larga, la sociedad se beneficia por me- dio de la ciencia aplicada y de la innovación tecnológica (Olivé, 2003, p. 7).

En el nuevo contrato social, por el contrario,

la ciencia y la tecnología no aparecen aisladas del resto de la sociedad, sino inmersas en ella, por lo que es imprescindible tomar en cuenta el papel de los funcionarios del Estado, de los industriales y empresarios, así como de los ciudadanos en general, pues en una sociedad democrática todos ellos deben sancionar positiva o negativamente el gasto público para desarrollarlas. Esta es una razón fundamental por la cual estos tres sectores -Estado, empresarios y ciudadanos- deben tener una mejor y más adecuada comprensión de los sistemas de ciencia de un país, de una región, o del mundo: qué son, cómo funcionan, por qué son importantes, y cómo pueden coadyuvar a la resolución de problemas y a satisfacer demandas sociales provenientes de sectores con distintos intereses (Olivé, 2003, p. 9).

En el viejo contrato social se definía una relación unidireccional entre ciencia, tecnología y sociedad: la sociedad apoyaba económica y políticamente a la ciencia y la tecnología, pero sin que tuviera derecho a intervenir en ellas. Como contrapartida, obtenía beneficios que garantizaban su progreso y mejores niveles de bienestar. En el nuevo contrato social la relación es bidireccional. La ciencia y la tecnología no aparecen aisladas, sino que hacen parte de un sistema de valores éticos, políticos, económicos, culturales y sociales mucho más amplio que las lleva a adquirir diferentes compromisos con la sociedad. A cambio, ciencia y tecnología reciben el apoyo de la sociedad, pero con la salvedad de que esta a su vez adquiere el derecho a intervenir en cuestiones científicas y tecnológicas cuando lo considere pertinente.

Para alcanzar las metas de este nuevo contrato social, debe garantizarse la participación de los ciudadanos, y esto exige una formación adecuada en temas de ciencia y tecnología. De ahí que la divulgación científica adquiera un papel fundamental en la configuración de estas nuevas relaciones. Ella permitiría formar ciudadanos con la competencia suficiente para participar de un modo idóneo en debates sobre ciencia y tecnología, y tomar así decisiones bien fundadas sobre distintos factores científicos y tecnológicos que los afectan directa e indirectamente (Castellanos, 2008; Cazaux, 2008; Lozano, 2008; Arias Cadavid, 2011; Perrault, 2013).

La divulgación científica sería, en definitiva, el medio más apropiado para garantizar la participación ciudadana y democratizar el conocimiento científico y tec- 
nológico. Pero para ello se insiste en la necesidad de abandonar un modelo deficitario de divulgación científica, y promover en su lugar un modelo democrático.

Esta tendencia a contraponer modelos deficitarios y modelos democráticos de divulgación científica suele justificarse en las perspectivas abiertas por el nuevo contrato social, y puede formularse de la siguiente manera: mientras el viejo contrato social implicaba un modelo deficitario, el nuevo contrato social implica un modelo democrático, que no es deficitario.

En este artículo defiendo la tesis de que dicha contraposición está mal fundada por dos razones. En primer lugar, el nuevo contrato social es compatible con diversos modelos democráticos de divulgación científica, y estos modelos no son compatibles entre sí necesariamente. En segundo lugar, algunos de estos modelos democráticos pueden calificarse también como deficitarios. Esto deja abierta la pregunta de cómo entender la divulgación científica en el marco del nuevo contrato social sobre ciencia y tecnología.

Pero antes de abordar este problema, es importante hacer una aclaración terminológica. Pues si existe algún consenso entre los estudiosos de la divulgación científica es que no hay ningún consenso en absoluto sobre el término más adecuado para hablar del tema (Canguilhem, 1996; Cooter y Pumfrey, 1994; Lozano, 2005; Daza et al., 2006; Erazo Pesántez, 2007; Márquez Valderrama, 2013; Raichvarg, 2013).

Los términos se multiplican, y por ello se emplean indistintamente algunos como popularización científica, vulgarización científica, difusión de la ciencia, comunicación de la ciencia, divulgación tecnocientífica, periodismo científico, comunicación científica pública, comunicación social de la ciencia, comunicación pública de la ciencia y la tecnología, apropiación social del conocimiento, apropiación social de la ciencia, la tecnología y la innovación y otros similares. En todos los casos, se reconoce que estos términos pertenecen al mismo universo lingüístico y conceptual, pero con cada uno de ellos se pretende ser más preciso que con los demás al tratar de determinar los elementos de ese universo. En otras palabras, el desacuerdo terminológico no parece reflejar un desacuerdo conceptual fundamental sobre el proceso comunicativo que se intenta describir.

Dada esta variedad terminológica, tomo el término divulgación científica como un hiperónimo de todos los demás. Y para ello asumo una postura basada en tradiciones wittgensteinianas y quineanas de filosofía del lenguaje, de acuerdo con la cual el significado de un término viene determinado por su uso, y no por alguna característica específica del mundo exterior. Por eso no considero que ninguno de los términos de ese universo lingüístico sea intrínsecamente deficitario, participativo, democrático, o cualquier otro calificativo similar, como se da a entender en ocasiones sobre algunos de ellos. Más bien los términos tendrán usos deficitarios, democráticos o participativos, y eso les dará un significado particular.

Así que emplearé divulgación científica como un hiperónimo, pero el lector podrá reemplazarlo siempre por su término favorito. En ningún caso, eso afectará el argumento principal que quiero desarrollar en este artículo.

\section{MODELOS DE DIVULGACIÓN CIENTÍFICA Y EL PROBLEMA DEL DÉFICIT}

Lewenstein (2003 y 2010) plantea que hay diversos problemas vinculados con la divulgación científica, y ellos tienen que ver con asuntos como qué significa hacer divulgación científica y quién la hace o debe hacerla, qué metas tiene, quién se beneficia o se perjudica, entre otros. Con el fin de resolver estos problemas, se han formulado diversos modelos comunicativos de divulgación científica, que él sistematiza en cuatro grandes grupos.

El primero es el modelo del déficit, que se caracteriza por asumir que el público tiene un déficit de conocimiento sobre temas de ciencia y tecnología, y propone que se diseñen e implementen programas para subsanarlo. El público aparece aquí como un barril vacío que es necesario llenar de conocimientos científicos y tecnológicos. Como contraparte, se ofrece la promesa de que una vez subsanado el déficit, las condiciones sociales serán mejores tanto para el sistema de ciencia y tecnología como para el público.

El segundo es el modelo del contexto que, aunque acepta que el público puede tener visiones incorrectas sobre diferentes aspectos de la ciencia y la tecnología, niega al mismo tiempo que ese público sea algo así como un barril vacío. Más bien las personas responden a la información científica y tecnológica de acuerdo con los contextos particulares en que la reciben. Estos contextos pueden ser sociales, culturales, políticos, económicos e incluso psicológicos, y se cruzan permanentemente entre sí. Entonces no hay propiamente un déficit de conocimiento, pues las personas construyen su propia comprensión del conocimiento científico y tecnológico con base en los contextos específicos que atraviesan en un momento dado. La promesa aquí es que, si se tienen en cuenta 
estos contextos, las personas desarrollarán visiones más adecuadas de tal conocimiento.

El tercero es el modelo del experto profano (lay expert), que establece una diferencia fundamental entre dos tipos de experticia: la de los expertos y la de los profanos. Los primeros sin duda tienen conocimiento especializado en temas de ciencia y tecnología. Pero los segundos tienen también sus propias formas de conocimiento especializado, solo que este es un tipo de conocimiento local, no propiamente científico y tecnológico. Este es un conocimiento ubicado en situaciones socioculturales concretas, como el que puede adquirir una comunidad de agricultores sobre los riesgos de un insecticida a partir de su uso en campo. Desde este modelo se propone que este conocimiento local es tan relevante para una sociedad como el conocimiento científico y tecnológico, y en algunas circunstancias incluso mucho más. Por eso debe garantizarse el diálogo entre ambos tipos de conocimiento, de modo que el profano también tenga la oportunidad de decidir sobre temas de ciencia y tecnología a partir de su propia forma de experticia, al menos con respecto a los riesgos y beneficios que aquellas puedan traer a su comunidad.

Finalmente, el cuarto es el modelo de participación pública, que se enfoca en la necesidad de plantear acciones para mejorar la participación del público en el diseño de la política científica y tecnológica de los países, y con ello incrementar la confianza de las personas en ciencia y tecnología. Se espera que tales acciones den como resultado mayores índices de democratización de la ciencia y la tecnología, de modo que el control sobre estas pase de una élite reducida de científicos y políticos al público y la sociedad en general.

Lewenstein separa los dos primeros modelos de los dos últimos, y su justificación es que ambos grupos se enfrentan con problemas diferentes. El primer grupo se concentra en los beneficios que trae el conocimiento científico y tecnológico, y por ello su foco es el diseño de estrategias que generen una mayor comprensión de ese conocimiento por parte del público. En cambio, el segundo grupo se concentra en el desequilibrio que puede existir entre el sistema de ciencia y tecnología y el resto de la sociedad al tomar decisiones que afectan a ambos, y por ello su foco es el diseño de estrategias para que ambas partes estén en igualdad de condiciones en esa toma de decisiones.

Puesto en otros términos, el punto que quiere destacar Lewenstein con su análisis, y que resulta particularmente explícito en su artículo de 2010, es que la divulgación científica tiene dos dimensiones distintas, pero complementarias: una dimensión epistémica y una dimensión política.

La dimensión epistémica se ocupa de familiarizar al público con los contenidos y los procedimientos de la ciencia y la tecnología, lo que incluye aspectos teóricos y conceptuales, pero también históricos, filosóficos, sociológicos, religiosos, psicológicos, entre muchos otros más. El punto central del proceso divulgativo aquí es el funcionamiento de la ciencia y la tecnología, tanto en términos de sus contenidos y procedimientos, como de las circunstancias vividas por las personas que los llevan a cabo.

La dimensión política se ocupa, en cambio, de familiarizar al público con los procesos y mecanismos de participación ciudadana en la generación de dichos contenidos y procedimientos. El punto central del proceso divulgativo aquí es otro: si acaso es deseable que los ciudadanos incidan en ciencia y tecnología y cómo pueden hacerlo, tomando en cuenta sus propias problemáticas y sus concepciones del mundo, sus intereses socioeconómicos, los riesgos asociados a la ciencia y la tecnología, el diseño del estado y su política científica y tecnológica, entre otros factores. En otras palabras, la dimensión política se concentra en asuntos de poder y autoridad en temas de ciencia y tecnología.

Una vez se distinguen estas dos dimensiones, el problema del déficit se complejiza mucho más de lo que se reconoce habitualmente.

Por una parte, el déficit puede referirse al grado de conocimiento que tienen los ciudadanos sobre ciencia y tecnología. En este caso, los científicos son expertos y el resto de los ciudadanos conforma un público básicamente ignorante sobre asuntos científicos y tecnológicos. Y tal ignorancia puede explicarse de distintas formas: falta de acceso a ese conocimiento, condiciones contextuales particulares que obstaculizan su comprensión, mayor confianza e interés en conocimientos locales que en conocimientos científicos y tecnológicos, entre otras razones. Este sería un déficit de tipo epistémico.

Pero el déficit también puede referirse al grado de participación ciudadana en las decisiones sobre ciencia y tecnología. En este caso, independientemente del grado de conocimiento que tenga el público sobre estos temas, son solo los científicos (y los políticos) quienes pueden participar en la toma de decisiones sobre ellos, pues son los únicos con la idoneidad requerida en tales circunstancias. Aquí no resulta im- 
portante garantizar el derecho del resto de los ciudadanos a participar en ciencia y tecnología, porque sin importar cuánto puedan saber al respecto, simplemente su falta de idoneidad hace que su voz sea irrelevante en los procesos de toma de decisiones. Este déficit no sería de tipo epistémico, sino político.

Usar indistintamente el déficit epistémico y el déficit político ha llevado a no reconocer qué tan complejos y variados pueden ser en realidad los modelos deficitarios de divulgación científica. Pues el carácter deficitario de cada modelo depende del tipo de déficit que se tenga en mente. En otras palabras, es posible, por ejemplo, que uno sepa poco de ciencia y tecnología (déficit epistémico alto) y, sin embargo, le garanticen un alto grado de participación ciudadana en la toma de decisiones sobre estas (déficit político bajo). $\mathrm{O}$, al contrario, que uno sepa mucho de ciencia y tecnología (déficit epistémico bajo) y, sin embargo, le garanticen muy poca participación ciudadana en la toma de decisiones sobre ellas (déficit político alto). Así que no hay una correlación directa entre ambos tipos de déficit, como a veces se quiere dar a entender. Y por esa razón, si retomamos las categorías de Lewenstein, es fácil concluir que no solo el primero, sino cualquiera de los otros tres modelos puede calificarse también como deficitario, de acuerdo con el tipo de déficit que se use como referencia.

Piénsese en el modelo del experto profano. Este modelo busca poner en igualdad de condiciones a expertos y profanos en los procesos de toma de decisiones sobre ciencia y tecnología. Esto sin duda evitaría distintas formas de déficit político, pues se garantizaría que tanto los conocimientos científicos y tecnológicos de los expertos como los conocimientos locales de los profanos sean parte del proceso de toma de decisiones. Pero esta distinción radical entre conocimientos científicos y tecnológicos y conocimientos locales puede levantarse sobre una valoración positiva de ciertas formas de déficit epistémico. Pues en este caso, puede resultar positivo no solo destacar que ambos tipos de conocimiento pertenecen a esferas completamente distintas del saber, sino que además son esferas que en muchas ocasiones ni siquiera se intersecan. Y por eso no sería en absoluto relevante, al menos no bajo ciertas circunstancias particulares, que los profanos adquirieran conocimiento científico y tecnológico. Lo verdaderamente relevante sería que su voz se oyera en los debates sobre estos temas, incluso si no llegan a figurar como expertos en ellos, incluso si ni siquiera están al tanto de tales temas. Bajo este modelo, por ejemplo, la voz de una comunidad indígena, dado el conocimiento ancestral que posee de su territorio, debe oírse para decidir si se realiza una excavación arqueológica allí o no. Así se contrarresta el déficit político. Pero al mismo tiempo puede ser completamente irrelevante divulgar conocimiento arqueológico a esa comunidad, pues el interés podría ser, digamos, divulgar los mecanismos de participación en ciencia y tecnología que tiene dicha comunidad para evitar la excavación, y no propiamente los saberes sobre evolución humana a que apelan los arqueólogos para justificarla. Aquí podrían valorarse más los saberes locales de la comunidad indígena que los saberes científicos de los arqueólogos, y promover con ello alguna forma de déficit epistémico sobre ciencia y tecnología.

La posibilidad de que existan este tipo de modelos, es decir, modelos que atacan el déficit político mientras promueven algún tipo de déficit epistémico, ha llevado a Dickson (2005, 27 de junio) a reivindicar nuevas versiones del modelo deficitario que fortalezcan el diálogo democrático, pero con base en la divulgación de información científica y tecnológica de la cual carecería el público. Dickson respalda así la idea de diferenciar las dimensiones política y epistémica de la divulgación científica, con la consecuencia de que formular un modelo que sea deficitario con respecto a una de las dos dimensiones (la epistémica en su caso) no implica que también tenga que serlo con respecto a la otra necesariamente (la política). Lewenstein (2010) no se opone del todo a estos esfuerzos, y en ocasiones incluso los alienta, mientras que Cortassa (2010 y 2016) y Meyer (2016) ponen el énfasis en el carácter eminentemente político que tienen las referencias a un déficit en la divulgación científica. Por su parte, Sturgis y Allum (2004) ven el modelo del déficit y el modelo contextual como complementarios, y por eso proponen integrarlos, pero tomando en cuenta tanto los factores epistémicos como los factores políticos de la divulgación científica. Ellos concluyen que la actitud del público hacia la ciencia y la tecnología variará de acuerdo con una relación proporcional entre estos dos tipos de factores.

En suma, la contraposición entre modelos deficitarios y modelos democráticos está mal fundada porque no solo hay distintas versiones del modelo deficitario, sino que además hay distintas versiones del modelo democrático, algunas de las cuales pueden calificarse asimismo como deficitarias. En otras palabras, la oposición entre déficit y democracia no es más que una falsa dicotomía. A continuación, quiero ahondar en esta tesis. 


\section{EL MODELO DEFICITARIO DEL VIEJO CONTRATO SOCIAL}

La tesis del nuevo contrato social se apoya en al menos dos presupuestos complementarios:

- El viejo contrato social se levanta sobre un modelo lineal de desarrollo científico y tecnológico, y este modelo de desarrollo implica un modelo deficitario de divulgación científica.

- El nuevo contrato social se levanta sobre un modelo alternativo de desarrollo científico y tecnológico, y este modelo de desarrollo implica un modelo democrático de divulgación científica, que no es deficitario.

El primer presupuesto no resulta particularmente problemático, como espero mostrar en esta sección, pero el segundo es difícil de aceptar, como espero mostrar en la próxima.

El modelo de desarrollo científico y tecnológico asociado con el viejo contrato social suele describirse como lineal. Esta línea de desarrollo comenzaría en la ciencia básica, y la idea sería que cuanto mayor sea la inversión en ciencia básica, mejores serán los productos obtenidos a partir de ella. Estos productos serían el insumo principal para realizar investigaciones en ciencia aplicada, las cuales a su vez servirían de base para las investigaciones en ingeniería. A partir de esta última, se generarían procesos de investigación y desarrollo (I+D) que derivarían en innovaciones tecnológicas y repercutirían en incrementos significativos de los niveles de bienestar social de los ciudadanos.

Para que este modelo de desarrollo funcione, se asume que la sociedad debe respaldar financiera y políticamente el sistema de ciencia y tecnología, pero sin interferir con él, precisamente porque son los científicos (de la academia y la industria), con ayuda de algunos políticos, quienes saben cómo funciona y cómo debe funcionar este sistema. El modelo emplea así una versión del triángulo de Sábato o modelo de la triple hélice, de acuerdo con el cual el desarrollo científico y tecnológico de los países depende de la intervención conjunta de tres actores: gobierno, academia e industria. De esa forma, se respalda la idea de que solo la academia y la industria, representadas por sus científicos, y el gobierno, representado por sus políticos, deben intervenir en asuntos de ciencia y tecnología. La sociedad tiene la responsabilidad de ofrecerles respaldo, pero no debe esperar ni exigir participación en tales procesos, pues carece de los conocimientos requeridos para hacerlo.
Este modelo lineal de desarrollo científico y tecnológico lleva de ese modo a un modelo deficitario de divulgación científica. Pues aquí se asume la existencia de un déficit epistémico que resulta importante subsanar mediante procesos comunicativos, pero solo con el fin de que haya cada vez mayor grado de respaldo social a la ciencia y la tecnología. Sin embargo, el modelo no postula que los ciudadanos carezcan de otras formas de conocimiento. Los ciudadanos no son tabulas rasas, libres de cualquier forma de conocimiento. Por el contrario, ellos tienen un gran número de conocimientos a su disposición. El problema es que esos conocimientos no pueden calificarse como científicos y tecnológicos, y por eso son irrelevantes en términos de participación ciudadana en ciencia y tecnología. Así que no se busca incrementar los niveles de participación ciudadana porque incluso aquellos ciudadanos bien informados sobre ciencia y tecnología aparecerán siempre como aficionados, y no como expertos en estos temas.

Es, pues, claro que hay aquí un modelo deficitario. Pero el análisis a partir de las dos dimensiones de la divulgación científica resulta mucho más informativo que aquellos análisis que se contentan con denunciar una oposición insalvable entre déficit y democracia, o alguna dicotomía similar. Pues este análisis permite comprender dos cosas. Por una parte, que la meta última del modelo de divulgación científica asociado con el viejo contrato social es sin duda terminar con el déficit epistémico. Pero, por otra parte, que esto se hace sin la pretensión de alterar en absoluto el déficit político que pueda tener el público en los procesos de toma de decisiones en ciencia y tecnología.

\section{EL DÉFICIT Y LOS MODELOS DEMOCRÁTICOS EN EL NUEVO CONTRATO SOCIAL}

A diferencia del viejo contrato social, el nuevo contrato tiene como una de sus principales aspiraciones romper el triángulo de Sábato, e incluir a la sociedad como una variable más para comprender cómo funciona y cómo debe funcionar el sistema de ciencia y tecnología. En esta nueva concepción, los cuatro componentes (academia, industria, gobierno y sociedad) tienen el derecho y la responsabilidad de intervenir en el sistema. Pero esto genera dificultades en dos direcciones. En primer lugar, se hace necesario redefinir el modelo de desarrollo científico y tecnológico. $Y$ esto lleva, en segundo lugar, a una redefinición del modelo de divulgación científica asociado con él, que se ha querido ver como democrático.

El inconveniente, sin embargo, es que al mismo tiempo se ha asumido que existe una oposición es- 
tricta entre modelos democráticos y modelos deficitarios. Y esto ha llevado a pasar por alto el hecho de que en realidad existen modelos democráticos que al mismo tiempo pueden calificarse como deficitarios. $Y$ creo que hay dos razones para esto.

La primera tiene que ver con la noción de participación, que se ha tomado como una condición necesaria y suficiente para distinguir entre déficit y democracia. Necesaria, porque se piensa que cuando se habla de democracia intrínsecamente se apela a alguna forma de participación, y esto no sucedería en el caso del déficit. Y suficiente, porque se asume que incluir la participación en la divulgación científica garantiza de inmediato un rol activo del público frente a la ciencia y la tecnología. Sin embargo, esta manera de ver la participación y su relación con la democracia olvida que los mecanismos de participación democrática son variados, e incluyen desde el simple hecho de dar un voto por una de varias opciones hasta la oportunidad de debatir de un modo continuo y prolongado sobre un tema particular con el fin de tomar decisiones sobre él. Ortegón Quiñones (2008), por ejemplo, incluye dentro de los tipos de participación no solo el control ciudadano y el empoderamiento, sino también el poder delegado, el apaciguamiento, la apatía, la manipulación, el clientelismo, la extorsión y el soborno, entre otros. En otras palabras, la participación democrática, en ciencia y tecnología o en cualquier otro ámbito, puede presentar diversas formas, y aunque no todas ellas se sustentan en un rol activo y crítico por parte de los ciudadanos, todas ellas pueden calificarse igualmente como democráticas.

Este último aspecto lleva a la segunda razón para explicar por qué se ha pasado por alto que algunos modelos democráticos de divulgación científica puedan ser deficitarios, y es que no se suele prestar atención al hecho simple, pero fundamental, de que no hay un único modelo (o sistema) de democracia, sino varios. Tales modelos de democracia pueden ser de tres tipos básicos: de participación directa, representativos o deliberativos, y las diferencias entre ellos se fundan precisamente en concepciones divergentes sobre qué es y cómo debe darse la participación ciudadana en los procesos de toma de decisiones en una democracia. En consecuencia, cuando se diseña un modelo democrático de divulgación científica en realidad no se hace más que reflejar un compromiso particular con determinado modelo de democracia, solo que aplicado al caso concreto de las relaciones entre ciencia, tecnología y sociedad. Y este es el punto que los proponentes de modelos democráticos no suelen resaltar en sus análisis.
Aunque más abajo no me refiero abiertamente a Held (2006) en ningún lugar, en esta sección me apoyo en su exposición sobre modelos de democracia. Con ella en mente, examino las propuestas de Olivé (2003), Lozano (2005 y 2008), Cuevas (2008) y Cortassa (2010 y 2016) como ejemplos paradigmáticos de modelos democráticos de divulgación científica. Mi interés es mostrar que estos modelos de divulgación científica reflejan compromisos claros con los tres tipos de modelos de democracia que acabo de mencionar, a saber: de participación directa, representativos y deliberativos. Y tales compromisos, sumados a mi análisis sobre la dimensión epistémica y la dimensión política de la divulgación científica, permiten identificar el carácter deficitario de algunos de ellos.

\subsection{Modelos de participación directa}

Olivé y Lozano insisten en el carácter participativo de sus modelos, pero no explican en realidad en qué consiste ese carácter exactamente. Aquí quiero mostrar que sus modelos pueden comprenderse mucho mejor si se interpretan desde la perspectiva de modelos de participación directa. El problema es que, a pesar de sus esfuerzos explícitos por alejarse del déficit, especialmente en el caso de Lozano, el diseño de sus modelos sobre la base de una participación directa del público en ciencia y tecnología les lleva a reafirmar posturas deficitarias. Empezaré, entonces, por comentar el modelo de Olivé, que denomino modelo de contribuyentes, y luego pasaré a comentar el modelo de Lozano, que se apoya en el de Olivé, pero introduciendo otros factores. Denomino a este último el modelo de la expresión de las libertades.

\subsubsection{El modelo de contribuyentes}

Tanto Olivé como Lozano enmarcan su discusión de la divulgación científica en términos del viejo y el nuevo contrato social. Olivé plantea en este sentido que el modelo de desarrollo científico y tecnológico asociado con el nuevo contrato social se caracteriza porque ciencia básica, ciencia aplicada, ingeniería e investigación y desarrollo (I+D) interactúan todas entre sí y todas con la sociedad simultáneamente, y solo así se produce bienestar social. No hay, pues, un orden o una secuencia particular que el desarrollo científico y tecnológico deba tomar en este modelo, a diferencia de lo que sucedía en el viejo contrato social, donde se postulaba una línea de desarrollo que comenzaba en la ciencia básica, avanzaba a la ciencia aplicada, luego a las investigaciones en ingeniería, y de allí a los procesos de I+D, que derivaban en innovaciones tecnológicas y bienestar social. En el nuevo contrato social, 
investigaciones en I+D, por ejemplo, pueden dar lugar a investigaciones en ciencia aplicada o en ingeniería, una opción que resultaría imposible desde la perspectiva del viejo contrato social.

Esta falta de orden o secuencia estricta en la producción de conocimiento en el nuevo contrato social se refuerza porque, de acuerdo con Olivé, la sociedad siempre tiene derecho a exigir reformulaciones en la ruta tomada para una investigación científica y tecnológica, no importa a cuál de las cuatro esferas (ciencia básica, ciencia aplicada, ingeniería e I+D) pertenezca. Es decir, la sociedad tiene derecho a intervenir en los procesos de producción de conocimiento cuando le parezca más conveniente, sin importar en qué esfera particular se dé esa producción. Y la justificación para esto es que las investigaciones en ciencia y tecnología se financian con dinero de los ciudadanos, que funcionan como contribuyentes. En esa medida, la sociedad tiene el derecho a decidir si se hacen investigaciones de, por ejemplo, ingeniería, pero no de ciencia básica, pues esto se hace con su dinero. Y ese no debería ser un argumento para detener el desarrollo científico y tecnológico en ningún momento. En última instancia, son los ciudadanos, como contribuyentes, quienes definen el modelo de desarrollo científico y tecnológico que se da aquí.

La principal consecuencia de tal modelo de desarrollo es la necesidad de contar con ciudadanos bien informados sobre ciencia y tecnología, pues de otro modo sus decisiones acerca de qué investigaciones financiar podrían estar mal fundadas, y esto podría repercutir negativamente en los procesos mismos de desarrollo científico y tecnológico. Para satisfacer tal necesidad, Olivé propone un modelo de divulgación científica que se levanta sobre dos aspectos complementarios.

El primer aspecto es la formación de mediadores expertos en diferentes áreas. En primer lugar, expertos en ciencias naturales, sociales y humanas que comprendan que el sistema de ciencia y tecnología debe satisfacer las demandas que le plantea la sociedad. En segundo lugar, expertos en comunicación pública de la ciencia y áreas afines, que sirvan de interfaz entre el sistema de ciencia y tecnología y la sociedad. Finalmente, expertos en estudios sobre ciencia, tecnología y sociedad (CTS), que tengan la capacidad de ver el sistema científico y tecnológico y sus relaciones con la sociedad desde perspectivas cada vez más amplias. Todo este proceso de formación de expertos tiene como finalidad garantizar la participación ciudadana en ciencia y tecnología por medio de la formación del público.
El segundo aspecto del modelo de Olivé es la autonomía epistémica de las comunidades científicas y tecnológicas. Esto no significa que tales comunidades estén al margen de la sociedad, pues de acuerdo con Olivé la justificación para financiar el sistema de ciencia y tecnología con fondos públicos es precisamente su capacidad para satisfacer las demandas de esa sociedad. Más bien la autonomía epistémica se refiere a que, al igual que sucedía en el viejo contrato social,

este modelo sigue sosteniendo la necesidad de la 'autonomía epistémica' de las comunidades científicas y tecnológicas, pues solo así pueden lograr sus objetivos en la producción de conocimiento. La autonomía epistémica significa que el conocimiento científico y sus aplicaciones son generados dentro de comunidades científicas y tecnológicas por medio de prácticas, es decir, de complejos de acciones que tienen sus propios y específicos valores y normas para organizar el trabajo, así como para la aceptación y rechazo de sus productos (hipótesis, teorías, modelos, técnicas, tecnologías) (Olivé, 2003, p. 9).

El punto es que, aunque se rechaza la autonomía política de las comunidades científicas y tecnológicas, se respeta aún su autonomía epistémica. Se produce así un blindaje de las comunidades científicas y tecnológicas frente a la incidencia y el control de otros actores sociales sobre ellas. Y la razón es que en última instancia son esas comunidades, y no el resto de comunidades que conforman una sociedad, las únicas que tienen la capacidad no solo de producir conocimiento científico y tecnológico, sino además de juzgar adecuadamente qué puede o no contar como tal para luego divulgarse.

El modelo democrático de Olivé se opone, en suma, a la presencia de cualquier forma de déficit político en las relaciones entre ciencia, tecnología y sociedad. Lo más importante es garantizar la participación ciudadana en estos temas. Y la participación tiene aquí un carácter directo, pues los ciudadanos funcionan como contribuyentes que tienen la potestad de decidir de forma inmediata en qué investigaciones debe o no gastarse su dinero.

Pero tal rechazo al déficit político se consigue a costa de reforzar el déficit epistémico de la sociedad en temas de ciencia y tecnología. Los tres tipos de mediadores expertos que propone su modelo, más la tesis de la autonomía epistémica, no hacen sino afianzar la idea de que el conocimiento de las comunidades científicas y tecnológicas y el conocimiento local de otras comunidades constituyen esferas del saber que bási- 
camente no se intersecan en ningún punto. Al igual que sucede con el modelo deficitario asociado con el viejo contrato social, este modelo democrático se apoya en la idea de que estas últimas comunidades no producen conocimiento científico y tecnológico, sino otras formas de conocimiento. ¿Y quiénes deciden que esas son otras formas de conocimiento? Precisamente las comunidades científicas y tecnológicas, cuyos procesos de producción de conocimiento resultan así blindados con respecto a los procesos que pueden llevarse a cabo en otras comunidades diferentes.

El rol de los mediadores expertos es así evitar el surgimiento de cualquier tipo de déficit político, de modo que los ciudadanos, en tanto contribuyentes, puedan ejercer su derecho democrático a participar directamente en la toma de decisiones sobre qué tipo de investigaciones respaldar. Pero para ello deben asumir la existencia de un déficit epistémico, que se levanta sobre el hecho de que la sociedad debe respetar la autonomía epistémica de las comunidades científicas y tecnológicas, lo que impide que al mismo tiempo pueda tener una participación directa en la producción y en la circulación de ese conocimiento. Las decisiones sobre estos últimos aspectos dependen exclusivamente de la autonomía epistémica de las comunidades científicas y tecnológicas.

\subsubsection{El modelo de la expresión de las libertades}

Mónica Lozano comparte varios elementos con León Olivé respecto a la lectura que ambos hacen del viejo y del nuevo contrato social, pero se aleja de él en un punto fundamental. Ella vincula el nuevo contrato social con un modelo de desarrollo científico y tecnológico inspirado en la tesis de Amartya Sen sobre las libertades humanas. Este cambio es significativo porque la justificación para la participación ciudadana en ciencia y tecnología no es ya la incertidumbre sobre qué hacer con el dinero de los contribuyentes, sino la expansión de las libertades humanas.

El punto es que, desde esta perspectiva, los ciudadanos tienen el derecho y la responsabilidad de elegir qué entienden por una vida digna, pues esta es un reflejo de la expresión de sus propias libertades. Y parte de esta elección recae sobre temas de ciencia y tecnología. La razón es que la ciencia y la tecnología se presentan en las sociedades contemporáneas como opciones que es necesario conocer y evaluar con el fin de elegirlas o no como un componente más de lo que cada uno entiende por una vida digna. Por supuesto, no todos tienen que incluirlas en sus vidas. Pero aquellos que decidan hacerlo deben tener la capaci- dad de evaluar los riesgos y los beneficios asociados con ellas, para poder así expresar adecuadamente sus libertades. Por eso el desarrollo científico y tecnológico se elabora aquí desde los intereses de la sociedad, y con una participación directa permanente en temas científicos y tecnológicos. Porque la sociedad se convierte no en un paciente, sino en un agente de desarrollo, que decide qué rutas deben tomar la ciencia y la tecnología, según lo que cada sociedad haya definido como una vida digna para sus miembros.

Este es, entonces, un modelo de desarrollo que debe garantizar la participación directa de los ciudadanos en la toma de decisiones sobre ciencia y tecnología, ya que de otro modo no es posible que ciencia y tecnología entren a jugar un rol verdaderamente efectivo en la vida de una persona como expresiones de su propia libertad, y no como imposiciones externas. Pero la única manera de garantizar tal participación directa es mediante procesos eficaces de divulgación científica que permitan a los ciudadanos tomar partido en los debates, no de forma arbitraria, sino como una manera informada de alcanzar la vida digna que todos ellos aspiran a tener para sí mismos.

Así que la divulgación científica en el modelo de Lozano contribuye a evitar el déficit político de los ciudadanos con respecto a la toma de decisiones sobre ciencia y tecnología. Puesto que lo más importante es que ellos puedan elegir qué debe o no incluirse en su vida, de modo que esta adquiera un estatus de dignidad, no puede recurrirse a intermediarios en ese proceso de toma de decisiones. Es un derecho y una obligación participar directamente en temas de ciencia y tecnología. Pero este rechazo al déficit político se hace una vez más a costa de trazar una línea insalvable entre el conocimiento científico y tecnológico, que producen los científicos al amparo de sus comunidades de especialistas, y otras formas de conocimiento, que producen los demás ciudadanos, y que no pueden calificarse en realidad como conocimientos científicos y tecnológicos.

De un modo similar a lo que sucede en el modelo deficitario del viejo contrato social y en el modelo de contribuyentes con la noción de autonomía epistémica, en el modelo de la expresión de las libertades solo se toma como conocimiento científico y tecnológico aquel tipo de conocimiento que se produce al amparo de un contexto institucional determinado, que lo valida y lo respalda como tal. Ese es el conocimiento que hace parte de los procesos de divulgación científica, y así se garantiza una vez más el blindaje de las comunidades científicas y tecnológicas frente a la in- 
cidencia y el control de otros actores sociales sobre ellas. Pues de acuerdo con Lozano, las demás formas de conocimiento producidas por el resto de los ciudadanos por fuera de esos contextos institucionales pueden reconocerse a lo sumo como "diferentes tipos de conocimiento y experticia" (Lozano, 2005, p. 70), pero nunca como conocimiento científico y tecnológico genuino. Por ello no hacen parte del proceso de divulgación científica, al menos no como formas de conocimiento científico y tecnológico, sino más bien como contrapartes de ese conocimiento. Porque no han sido producidas y avaladas como tal por comunidades científicas en contextos institucionales específicos. Arias Cadavid (2011, p. 162), que sigue de cerca el modelo de Lozano, resume muy bien este punto cuando afirma que "el modelo democrático reconoce la existencia de múltiples tipos de conocimiento -en ocasiones en conflicto-, y trata de consensuarlos a través del debate público abierto y constructivo".

La eliminación del déficit político se levanta, en suma, sobre la reafirmación de un déficit epistémico de la sociedad acerca de temas científicos y tecnológicos. No se niega que la sociedad tenga algún tipo de conocimiento. Se niega más bien que esta tenga conocimiento científico y tecnológico. Y por ese motivo la divulgación científica tendría como una de sus funciones entrar a mitigar tal déficit epistémico, aparentemente mediante la puesta en marcha de algo así como un diálogo de saberes entre conocimientos científicos y tecnológicos y otros conocimientos que no podrían calificarse como tales. A pesar de toda la formación que hayan podido recibir sobre ciencia y tecnología en instituciones como la escuela, la universidad o los medios de comunicación, este modelo postula que los ciudadanos carecen de conocimientos científicos y tecnológicos, aunque no de otros tipos de conocimiento, y por tanto la divulgación científica debe entrar a suplir tal carencia mediante un diálogo que se apoye en estos últimos. Solo así puede garantizarse que los ciudadanos tengan el fundamento suficiente para participar directamente en la toma de decisiones sobre qué aspectos de la ciencia y la tecnología desean incluir en su concepción de una vida digna.

\subsection{Modelos representativos}

Los modelos de participación directa que acabo de presentar se caracterizan por ofrecer diversas opciones para atacar el déficit político, pero al hacerlo caen en alguna forma de déficit epistémico. La razón, me parece, tiene que ver con las dificultades propias de apelar a cualquier forma de participación directa en temas de ciencia y tecnología. Pues, aunque resulte plausible concebir la participación directa del público en asuntos de diseño de política científica, sea por cuestiones monetarias o de algún otro tipo, es mucho más difícil aceptar que el público pueda o incluso deba participar directamente en la producción de conocimiento científico y tecnológico. Es plausible que el público decida, por ejemplo, si financia o no una investigación sobre células madre. Pero, ¿̇esperamos que ingrese también al laboratorio y tome directamente decisiones sobre qué procedimientos seguir, qué experimentos diseñar, o qué criterios emplear para determinar si algo cuenta o no como evidencia empírica? La respuesta parece ser negativa. Y quizá esto explica que se apele en este punto a nociones como las de autonomía epistémica y diferentes tipos de conocimiento y experticia para justificar la exclusión de la sociedad en estos procesos. En ambos casos parece razonable defender una perspectiva en la cual se garantice la participación directa en el diseño de política científica y tecnológica, pero de ningún modo en los procesos mismos de producción de conocimiento.

Pero si entonces parece claro que la participación directa no puede o no debe garantizarse con respecto a la producción de conocimiento, ¿no debería también dejarse en duda este tipo de participación con respecto al diseño de política científica? Después de todo, si el público no sabe realmente sobre temas de ciencia y tecnología, ¿cómo estar seguros de que tomará decisiones adecuadas sobre el control político de la ciencia y la tecnología? ¿No sería más razonable en realidad que otros tomen las decisiones por él? Así llegamos precisamente a los modelos representativos de divulgación científica, y para ello me voy a apoyar en el tratamiento que hace Ana Cuevas de las relaciones entre conocimiento científico, democracia y ciudadanía.

Cuevas enmarca su aproximación a la divulgación científica de una forma un poco distinta a Olivé y Lozano. Como ellos, Cuevas empieza por notar la situación de desconfianza general hacia la ciencia y la tecnología que caracterizó a varios sectores sociales en la segunda mitad del siglo XX, y que llevó finalmente a posturas como la tesis del nuevo contrato social. Pero para ella el problema de fondo es cómo lograr que las relaciones entre el sistema de ciencia y tecnología y la sociedad sean más fluidas, comprensivas y receptivas. Su solución a este interrogante envuelve dos aspectos: sensibilización de científicos y políticos y formación de ciudadanos con mejores conocimientos científicos y tecnológicos. El propósito del segundo aspecto es conseguir mayor participación ciudadana en las decisiones sobre ciencia y tecnología, con respecto 
tanto a la determinación de sus objetos de investigación como al grado de financiación pública que deben recibir. Para alcanzar este propósito, Cuevas rechaza el modelo deficitario tal como lo describe Durant (1999), y se enfoca en el modelo democrático.

Sin embargo, en este punto ella formula una pregunta que revela la complejidad del problema que tiene en frente, a saber: ¿qué tan participativo debe ser el modelo democrático con que se ha de formar a los ciudadanos para que intervengan en ciencia y tecnología? Su respuesta se apoya en el análisis del debate entre Dewey y Lippmann en la primera mitad del siglo XX sobre las condiciones de participación ciudadana en los estados democráticos. Ella extiende sus conclusiones sobre este debate al caso particular de los modelos democráticos de divulgación científica, y muestra que es posible diseñar por lo menos dos modelos democráticos diferentes. En esta sección me ocupo del modelo de élites, inspirado en Lippmann, y que Cuevas no respalda. Más abajo volveré sobre el modelo deliberativo, inspirado en Dewey, y que Cuevas sí respalda.

El modelo de élites se funda en una constatación empírica difícil de rebatir: las sociedades contemporáneas contienen un alto grado de complejidad y especialización en casi todos sus temas, y por eso es difícil esperar que sus ciudadanos estén en la capacidad suficiente para ser expertos en todos ellos al mismo tiempo. No puede exigírseles, por tanto, que tengan la competencia necesaria para tomar decisiones bien informadas en un amplio número de asuntos que los afectan directa e indirectamente. Por el contrario, en muchos casos ellos comprenden la realidad social y política con base en estereotipos y prejuicios, debido precisamente a ese alto grado de especialización sobre el que se levantan sus sociedades. Incluso hay ocasiones en que ni siquiera están interesados en tales temas, y esta falta de interés es una opción perfectamente respetable y defendible en sociedades democráticas.

No parece, entonces, conveniente poner el peso de la toma de decisiones sobre un gran número de temas, incluidos los científicos y tecnológicos, en los hombros de los ciudadanos. Incluso sería políticamente irresponsable basar tales decisiones en los puntos de vista de personas que o no están al tanto de estos temas, o no están interesadas en ellos, o ambos. Pero, aun así, todo estado democrático tiene la obligación de velar por el bienestar y el buen desarrollo de sus ciudadanos. Y por eso en principio debe garantizar que se tomen las mejores decisiones posibles acerca de cualquier asunto que sea o pueda llegar a ser rele- vante para esos ciudadanos, y esto incluye la ciencia y la tecnología. ¿Qué hacer entonces? ¿Cómo velar por ese bienestar y buen desarrollo, pero sin exigir a cambio una participación directa, como se sigue de los modelos de Olivé y Lozano, y sin renunciar al mismo tiempo a las instancias democráticas? La respuesta es un modelo democrático de élites.

En este modelo, los ciudadanos eligen a los representantes más idóneos para satisfacer sus demandas sociales, y estos conforman élites de expertos y líderes. Los primeros recaban información y coordinan investigaciones de relevancia para el resto de la sociedad, mientras que los segundos ejecutan decisiones políticas con base en la labor de los expertos. Así que el desarrollo científico y tecnológico depende directamente de las decisiones que tomen expertos y líderes. Y estos a su vez deciden qué divulgar a la sociedad sobre ciencia y tecnología, de acuerdo con lo que resulte más conveniente para ella desde un punto de vista político y epistémico. Por eso, aunque el modelo se levanta sobre la postulación de un déficit epistémico y parcialmente político de parte de los ciudadanos, continúa siendo democrático por dos motivos. No solo porque garantiza la participación ciudadana en la elección de representantes (expertos y líderes), sino porque además somete la ciencia y la tecnología al mismo tipo de control democrático que reciben otros componentes del estado en una democracia representativa.

El modelo de élites muestra, en suma, que es posible formular un modelo de divulgación científica que sea democrático y al mismo tiempo se levante sobre la postulación de un déficit epistémico y parcialmente político de los ciudadanos. Y digo parcialmente político, porque los ciudadanos conservan aún su derecho democrático a participar en la toma de decisiones sobre temas de ciencia y tecnología, solo que mediante la elección de sus representantes. Se garantiza, pues, la participación en ciencia y tecnología, pero tal participación no es ni epistémica ni políticamente directa. Todo esto sin renunciar a los objetivos trazados en el nuevo contrato social sobre la integración de la sociedad en la toma de decisiones democráticas sobre asuntos científicos y tecnológicos.

\subsection{Modelos deliberativos}

He mostrado en las dos secciones anteriores que, a partir de consideraciones de participación directa y representatividad, es posible diseñar modelos de divulgación científica que sean democráticos y deficitarios al mismo tiempo. Así que, contrario a lo que en general han querido dar a entender muchos defenso- 
res de la tesis del nuevo contrato social, el hecho de que un modelo de divulgación científica sea democrático no garantiza inmediatamente que no pueda ser también deficitario. El problema es más bien cómo formular un modelo de divulgación científica que sea democrático, pero no deficitario, pues no es del todo obvio que tal cosa pueda hacerse.

En este sentido, creo que los modelos deliberativos son la opción que muestra mejores resultados en esta dirección por el momento. Aquí quiero comentar en particular la versión desarrollada por Cuevas con base en su lectura de Dewey. Esta versión concuerda en líneas generales con el modelo que presenta Cortassa a partir de su crítica a la obsesión contemporánea con el déficit como categoría fundamental de análisis, y su propia propuesta de basar los modelos de divulgación científica en la existencia de asimetrías cognitivas (o asimetrías epistémicas, para usar mi propia terminología) que posibiliten la diseminación efectiva del conocimiento científico y tecnológico entre diferentes agentes.

En este modelo, los ciudadanos aparecen como sujetos autogobernados que tienen la capacidad de desarrollar hábitos inteligentes para la indagación. Pero estos son hábitos que se forman y fortalecen no en situaciones de aislamiento, sino a partir de su integración en procesos deliberativos con otros. La deliberación exige así el ejercicio de la libertad, entendida como la auto-dirección de un sujeto que comprende que sus acciones individuales tienen una dimensión moral. Y esta dimensión moral se manifiesta en el impacto de tales acciones en los demás.

Lo anterior lleva a plantear que el desarrollo de la ciencia y la tecnología no debe responder a intereses particulares ni de los expertos ni del resto de los ciudadanos. Exige más bien el trabajo mancomunado de ambos grupos. Y por eso la divulgación científica se vuelve relevante aquí, porque ella se concibe como un proceso deliberativo entre expertos y los demás ciudadanos, que no son expertos, pero que tampoco son completamente ignorantes sobre ciencia y tecnología, como se da a entender en los modelos de participación directa y representativos. Este proceso deliberativo, que se levanta sobre la asimetría existente entre el grado de conocimiento científico y tecnológico de los expertos y aquel de los demás ciudadanos, permite que estos últimos afiancen y amplíen los conocimientos científicos y tecnológicos que ya poseen, y esto contribuye a convertirlos en personas con mejor información sobre ciencia y tecnología, y a darles mayor poder político en la toma de decisiones. Pero asimismo transforma a los expertos en personas inte- resadas en las consecuencias sociales, ambientales y políticas de sus investigaciones.

Este modelo ataca, en definitiva, tanto el déficit epistémico como el déficit político, al intentar garantizar que los procesos deliberativos refuercen la participación ciudadana en ciencia y tecnología desde dos puntos de vista complementarios. No solo porque políticamente los ciudadanos tienen el derecho democrático a hacerlo, sino además porque el proceso deliberativo los convierte en sujetos epistémicamente fiables en estos temas, gracias al afianzamiento y la ampliación del conocimiento científico y tecnológico, tal vez aún incipiente, con que entraron al proceso deliberativo. Es decir, sujetos que, mediante su interacción con otros, mejoran y vuelven más sofisticado el conocimiento científico y tecnológico con que contaban al empezar la deliberación. Pero no se asume en ningún momento que los así llamados conocimientos locales no puedan calificarse a su vez como conocimientos científicos y tecnológicos. Siempre y cuando ambos tipos de conocimiento satisfagan los mismos criterios epistémicos, habrá que concluir que ambos pertenecen a la misma esfera del saber.

Los modelos deliberativos de divulgación científica generan, sin embargo, el mismo tipo de interrogantes que pueden surgir en el caso de su contraparte en los modelos de democracia. Por ejemplo, aunque con ellos se pretende que la deliberación se origina con algún grado de conocimiento de las partes sobre el tema a tratar, grado que crece en la medida en que la deliberación avanza, no se puede garantizar que no existan desequilibrios con respecto a asuntos como el poder de unos grupos sobre otros, el mejor dominio de un tema o incluso las habilidades retóricas para persuadir a los demás, entre otros. Esto significa que los resultados de la deliberación pueden llegar a viciarse por las características individuales y sociales de los actores que participan en ella. Y así de hecho existe la posibilidad de que ciertos grupos orienten todo el proceso deliberativo hacia sus propios intereses particulares. La asimetría epistémica puede convertirse, entonces, en asimetría política también, al menos si los actores se concentran únicamente en usar las estrategias que más convengan a su propia causa.

Otro aspecto para tomar en consideración es que no resulta del todo claro quién decide cuáles son los criterios epistémicos que algo debe satisfacer para contar como conocimiento científico y tecnológico. $Y$ esto es importante porque si se parte de la idea de que incluso antes de iniciar el proceso deliberativo el público tiene no solo otros tipos de conocimiento, 
sino además algún tipo de conocimiento científico y tecnológico, entonces cabe la pregunta de cómo se establece esto. ¿Acaso porque ciertas comunidades científicas y tecnológicas particulares lo deciden así o porque el público lo decide así? ¿O acaso porque pueden identificarse criterios universales de cientificidad previos a la existencia misma de comunidades particulares, sean estas científicas o no? Estas preguntas sugieren que, una vez más, la asimetría epistémica puede dar lugar a una asimetría política en el proceso deliberativo, en esta ocasión con respecto a quién decide qué habrá de considerarse o no como conocimiento científico y tecnológico.

De cualquier forma, y a pesar de los interrogantes que puedan surgir al momento de querer implementarlos, especialmente en lo que concierne a las asimetrías epistémicas que los soportan, los modelos deliberativos de divulgación científica parecen ofrecer una opción viable para el diseño de modelos democráticos que no sean deficitarios. Estos son modelos que no niegan la existencia de algún grado de conocimiento científico y tecnológico al público, conocimiento que puede mejorarse con la deliberación, y que establece un punto de partida para respaldar la participación política de ese público en la toma de decisiones con los expertos.

Los modelos deliberativos aparecen, por tanto, como la mejor opción para todos aquellos que quieren evitar la presencia del déficit en la divulgación científica. Pues son modelos que pueden calificarse, al mismo tiempo, como democráticos y no deficitarios. Y una afirmación semejante no parece ser posible en el caso de los modelos de participación directa y los modelos representativos.

\section{CONCLUSIÓN}

He querido mostrar en este artículo que no solo hay distintas versiones del modelo democrático de divul- gación científica, las cuales no siempre resultan compatibles entre sí, sino que además muchas de ellas pueden calificarse como deficitarias, sea desde una dimensión epistémica, desde una dimensión política, o desde ambas. Esto lleva a rechazar uno de los presupuestos fundamentales de la tesis del nuevo contrato social, a saber: que necesariamente el modelo democrático de divulgación científica no es deficitario.

A pesar de esto, mi propio punto de vista es que conviene seguir pensando las relaciones entre ciencia, tecnología y sociedad en el marco del nuevo contrato social, debido precisamente al papel activo que se atribuye aquí a la sociedad con respecto al sistema de ciencia y tecnología. Y no solo eso. Conviene además seguir asociando a este marco un modelo de divulgación científica que tenga un carácter democrático. El problema es formular ese modelo. Pues, como vimos, los modelos de participación directa y los modelos representativos, al menos en su estado actual, no logran evitar ciertas formas de déficit. Y los modelos deliberativos, aunque lo logran, dejan abiertos ciertos interrogantes sobre las asimetrías epistémicas y políticas que los soportan, interrogantes que aún no es del todo claro cómo resolver.

En cualquier caso, parece evidente que cualquiera sea el modelo de divulgación científica que se elija, este será en última instancia un reflejo de concepciones políticas mucho más amplias sobre la configuración de los sistemas democráticos en los estados modernos. Y en su concepción más básica estos pueden ser sistemas de participación directa, representativos o deliberativos. Tales sistemas se intentarán trasladar de un modo u otro al diseño de los sistemas de ciencia y tecnología de cada país, y esto incluye sus modelos de divulgación científica. Por tanto, apelar a la democracia en el debate, pero excluyendo al mismo tiempo la discusión sobre los modelos de democracia, como en general se ha hecho hasta ahora, parece abiertamente injustificable.

\section{BIBLIOGRAFÍA}

Arias Cadavid, L. M. (2011). La exclusión y la comunicación de la ciencia y la tecnología en un nuevo contrato social sobre la ciencia. Trilogía, 4, pp. 155-167. https:// doi.org/10.22430/21457778.148

Bush, V. (1945). Science, the Endless Frontier Washington: Government Printing Office.

Canguilhem, G. (1996). Necesidad de la 'difusión científica'. Sociología, 19, pp. 27-33.
Castellanos, P. (2008). Comunicar la ciencia en la sociedad del riesgo. Los medios y los museos de ciencias como mediadores sociales. Razón y Palabra, 13 (65). [En línea]. Disponible en: http://www.razonypalabra. org.mx/N/n65/actual/pcastellanos.html

Cazaux, D. (2008). La comunicación pública de la ciencia y la tecnología en la 'sociedad del conocimiento'. Razón y pala- bra, 13 (65). [En línea]. Disponible en: http://www.razonypalabra.org.mx/N/ n65/actual/dcasaux.html

Cetto, A. M. (ed.) (2000). World Conference on Science: Science for the Twenty-First Century. A New Commitment. Londres: UNESCO.

Cortassa, C. G. (2010). Del déficit al diálogo, ¿y después? Una reconstrucción crítica 
de los estudios de comprensión pública de la ciencia. Revista Iberomericana de Ciencia, Tecnología y Sociedad, 5 (15), pp. 117-124.

Cortassa, C. (2016). In science communication, why does the idea of a public deficit always return? The eternal recurrence of the public deficit. Public Understanding of Science, 25 (4), pp. 447-459. https:// doi.org/10.1177/0963662516629745

Cooter, R. y Pumfrey, S. (1994). Separate Spheres and Public Places: Reflections on the History of Science Popularization and Science in Popular Culture. History of Science, 32 (3), pp. 237-267. https:// doi.org/10.1177/007327539403200301

Cuevas, A. (2008). Conocimiento científico, ciudadanía y democracia. Revista lberoamericana de Ciencia, Tecnología y Sociedad, 4 (10), pp. 67-83.

Daza, S., Arboleda, T., Rivera, Á., Bucheli, V. y Alzate, J. F. (2006). Evaluación de las actividades de comunicación pública de la ciencia y la tecnología en el Sistema Nacional de Ciencia y Tecnología Colombiano, 1990-2004. Bogotá: Observatorio Colombiano de Ciencia y Tecnología.

Dickson, D. (2005, 27 de junio). The case for a 'deficit model' of science communication. SciDev.net. [En línea]. Disponible en: http://www.scidev.net/global/communication/editorials/the-case-for-a-deficit-model-of-science-communic.html

Durant, J. (1999). Participatory technology assessment and the democratic model of the public understanding of science. Science and Public Policy, 26 (5), pp. 313-319. https://doi. org/10.3152/147154399781782329

Erazo Pesántez, M. A. (2007). Comunicación, divulgación y periodismo de la ciencia. Una necesidad imprescindible para Iberoamérica. Quito: Planeta.
Gibbons, M. (1999). Science's new social contract with society. Nature, 402, pp. C81-C84. https://doi. org/10.1038/35011576

Guston, D. H. (2000). Between Politics and Science: Assuring the Integrity and Productivity of Research. Cambridge University Press. https://doi.org/10.1017/ CBO9780511571480

Held, D. (2006). Models of Democracy. Cambridge, UK, Malden, MA: Polity Press.

Kevles, D. J. (1995). The Physicists. The History of a Scientific Community in Modern America. Harvard University Press.

Lewenstein, B. V. (2003). Models of public communication of science and technology. [En línea]. Disponible en: http:// disciplinas.stoa.usp.br/pluginfile. php/43775/mod_resource/content/1/ Texto/Lewenstein\%202003.pdf

Lewenstein, B. V. (2010). Models of Public Understanding: The Politics of Public Engagement. ArtefaCToS, 3 (1), pp. 13-29.

López Cerezo, J. A., Méndez Sanz, J. A. y Todt, O. (1998). Participación pública en política tecnológica: Problemas y perspectivas. Arbor, 49 (627), pp. 279-308. https://doi.org/10.3989/arbor.1998. i627.1767

Lozano, M. (2005). Programas y experiencias en popularización de la ciencia y la tecnología: panorámica desde los países del Convenio Andrés Bello. Bogotá: Convenio Andrés Bello.

Lozano, M. (2008). El nuevo contrato social sobre la ciencia: retos para la comunicación de la ciencia en América Latina. Razón y Palabra, 65. Disponible en: http://www.razonypalabra.org.mx/N/ n65/actual/mlozano.html. Consultado el 15 de agosto de 2015.
Lubchenco, J. (1998). Entering the Century of the Environment: A New Social Contract for Science. Science, 279 (5350), pp. 491-497. https://doi.org/10.1126/ science. 279.5350 .491

Márquez Valderrama, J. (2013). ¿Vulgarización vs. degradación? Un análisis en perspectiva histórica. En: Domínguez Gómez, E., Echeverry Mejía, J. A. y Castaño Grajales, M. (eds.). Apropiación social del conocimiento. El papel de la comunicación. Medellín: Universidad de Antioquia, pp. 183-201.

Meyer, G. (2016). In science communication, why does the idea of a public deficit always return? Public Understanding of Science, 25 (4), pp. 433-446. https:// doi.org/10.1177/0963662516629747

Olivé, L. (2003). Por un nuevo contrato social sobre la ciencia y la tecnología. Ciencia y desarrollo 172, pp. 7-12.

Ortegón Quiñones, E. (2008). Guía sobre diseño y gestión de la política pública. Bogotá: Organización del Convenio Andrés Bello / Colciencias / Instituto de Estudios Latinoamericanos.

Perrault, S. (2013). Communicating Popular Science: From Deficit to Democracy. Palgrave Macmillan. https://doi. org/10.1057/9781137017581

Raichvarg, D. (2013). Principios de la divulgación de las ciencias. En: Domínguez Gómez, E., Echeverry Mejía, J. A. y Castaño Grajales, M. (eds.). Apropiación social del conocimiento. El papel de la comunicación. Medellín: Universidad de Antioquia, pp. 9-27.

Sturgis, P. y Allum, N. (2004). Science in Society: Re-Evaluating the Deficit Model of Public Attitudes. Public Understanding of Science, 13 (1), pp. 55-74. https://doi. org/10.1177/0963662504042690 